\title{
Use of energy dispersive method with tungsten carbide opposed anvil high pressure set-up
}

\author{
A K SINGH and S USHA DEVI \\ Materials Science Division, National Aeronautical Laboratory, Bangalore 560017 , India \\ MS received 14 April 1987
}

\begin{abstract}
The use of energy dispersive method has been discussed to record the X-ray diffraction data from a tungsten carbide opposed anvil high pressure set-up. It is pointed out that a proper collimation of the incident and the diffracted beams can eliminate the scattering from the gasket material. This results in an appreciable improvement in the quality of the recorded diffraction data. The choice of the various parameters has been discussed. The data on sodium chloride are presented in support of this analysis.
\end{abstract}

Keywords. High pressure studies: energy-dispersive method; beam divergence; gasket scattering.

\section{Introduction}

The energy dispersive method has been used extensively in recent years in recording $X$-ray diffraction data from specimens under high pressure. This method offers high speed of data collection and has been used mostly with diamond anvil cells. In this paper we have discussed the use of energy dispersive method in recording the diffraction data from tungsten carbide opposed anvil set-up. Freud and LaMori (1969), while discussing the use of an energy-dispersive technique with a split-die belt apparatus, suggested a configuration of the specimen, and the incident and the diffracted beams, which can eliminate the unwanted scattering from the gasket material. However, Freud and LaMori (1969) neither analysed this aspect in detail nor perform any experiments using the geometry they suggested. Since their geometry is also applicable to the tungsten carbide opposed anvil set-up, a detailed analysis has been presented in this paper. The high pressure data on sodium chloride gathered with a tungsten carbide opposed anvil set-up under the Freud-LaMori geometry are presented to support the analysis given in this paper.

\section{General considerations}

The working principle of a tungsten carbide anvil high pressure cell for X-ray diffraction work (Jamieson and Lawson 1962; Perez-Albuerne et al 1964; McWhan and Bond 1964) is shown schematically in figure 1. The powdered specimen is contained in a hole at the centre of a X-ray transparent gasket (boron, boron-epoxy, beryllium) typically $3 \mathrm{~mm}$ in diameter and about $0.2 \mathrm{~mm}$ thick. The specimen is pressurized by loading the gasket-specimen assembly between the tips of two tungsten carbide anvils. A collimated $x$-ray beam. normal to the direction of the applied load, passes through the gap between the anvils and illuminates the specimen. The commonly used angle dispersive method employs MoK radiation, and the diffraction pattern is recorded on a photographic film. The use of energy 


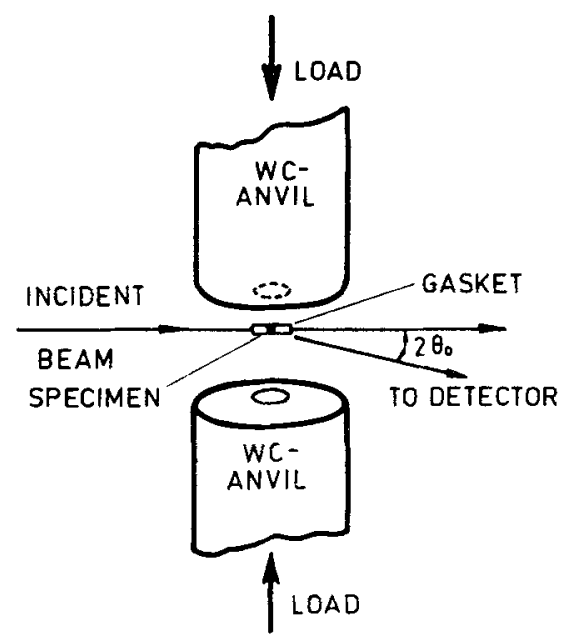

Figure 1. Working principle of a tungsten carbide opposed anvil set-up for X-ray diffraction work.

dispersive method discussed here requires white (continuous) radiation from either molybdenum or tungsten target. A solid state detector is placed at a fixed $2 \theta\left(=2 \theta_{0}\right)$ to detect the entire range of energy spectrum in the direction $2 \theta_{0}$. The diffraction peaks which appear at different energies are recorded simultaneously. The $d$-spacing of a set of planes with indices $(h k l)$ is given by

$$
d(h k l)=h c / 2 E \sin \theta_{0},
$$

where $E, h$ and $c$ are respectively the energy at the diffraction maximum, the Planck's constant and the velocity of light.

\section{Beam divergence}

The divergences (termed radial divergences) of the incident and the diffracted beams (in a plane perpendicular to the loading direction and passing through the centre of the gasket) along with the other parameters used in further discussion are marked on figure 2. The divergence of the incident beam, $\alpha_{i}$ and the dimension, $2 a$, of the specimen region at the centre, bathed by the incident $X$-ray beam are given by

$$
\begin{aligned}
& x_{i}=W_{i} / L_{i}, \\
& 2 a=\alpha_{i}\left(L_{i}+I_{i}\right) .
\end{aligned}
$$

The symbols appearing in (2) and (3) are explained in figure 2. The above equations are valid for the condition $2 r>\alpha_{i}\left(L_{i}+l_{i}\right)$ where $r$ is the radius of the specimen. It will become clear from the later discussions that the analysis under this condition alone is of interest. In deriving (2) and (3) it is assumed that the focal spot is larger than $u_{i}\left(D_{i}+L_{i}\right)$. This condition is satisfied if a conventional X-ray tube is used. However, microfocus or fine focus $X$-ray tubes provide focal spots which are $100 \mu$ or less in size. In such cases $x_{i}$ is smaller than the value given by (2). It can be easily shown that in this case $x_{i}$ is given by

$$
x_{i}=\left(I_{1}+H, 1\left(2 H_{1}+D, D_{1}\right)\right.
$$




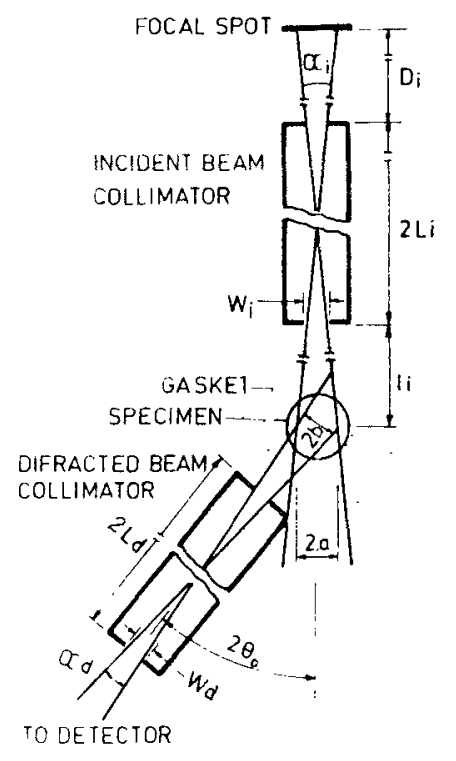

Figure 2. Arrangement for restricting the incident and the difiracted beam divergences.

where $f_{1}$ is the dimension of the focal spot in the plane of figure 2 , and $D_{i}$ is the distance between the focal spot and the nearest pinhole of the collimator.

The expressions for the radial divergence, $\alpha_{d}$, of the diffracted beam, and $2 b$, the width of the region (at the centre of the specimen) seen by the detector can be obtained by replacing suffix $i$ in (2) and (3) by $d$. The resulting expressions for $\alpha_{d}$ and $2 b$ are valid if the detector surface size is greater than $\alpha_{d}\left(L_{d}+D_{d}\right)$. This condition is always satisfied in practice.

The incident and the diffracted beams possess divergences also in the plane normal to the plane of figure 2 . These are termed axial divergences and are denoted by $\beta_{i}$ and $\beta_{d}$. The values of $\beta_{i}$ and $\beta_{d}$ depend on the gasket thickness, $t$, and the shape of the collimator pinhole. If the pinhole is circular and $t \geqslant 2 a$, then $\beta_{i}=\alpha_{t}$. For $t<2 a, \beta_{i}$ is given by

$$
\beta_{i}=\left(W_{i}+t\right) /\left(2 L_{i}+l_{i}\right)
$$

These results are valid only if the axial dimension of the focal spot is greater than $x_{i}\left(L_{i}+D_{i}\right)$. Similar consideration of the geonetry of the dilfracted beam collimator shows that for $t \geqslant 2 b, \beta_{d}=x_{d}$. For $t<2 b$, an expression for $\beta_{i}$ can be obtained by simply replacing suffix $i$ in (5) by $d$.

\section{Scattering from the gasket}

The orjgin of scattering from the gasket material in energy-dispersive mode of data collection can be understood from figure 3. Since the divergences of the incident and the diffracted beans are small (rarcly exceeding a few milliradians). the reglons of the gasket and the specimen illuminated by the incident beam can be considered to be hound by pallalliwes. $1 B$ and ( $T$ ). the ditance between the two being 2a. Similarly, the illuminated region from whes the sartered indion reaches the derector is 


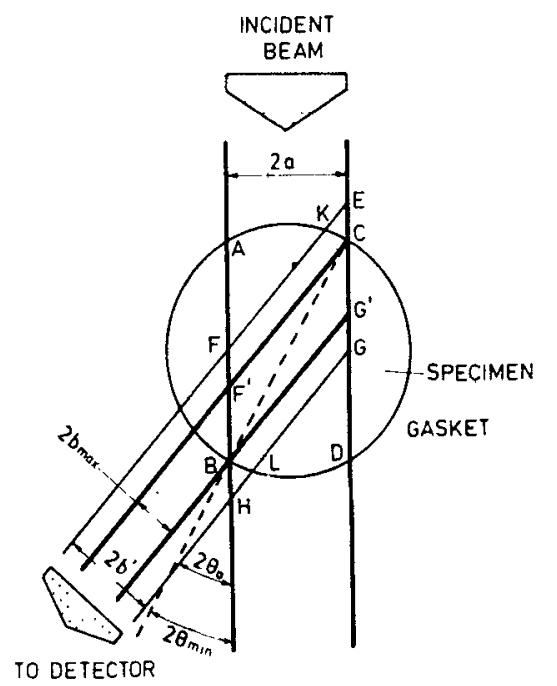

Figure 3. Origin of the scattering from the gasket material. The scattered radiation from the gasket region ECK and $\mathrm{LHB}$ reaches the detector.

bound by parallel lines EF and GH separated by $2 b^{\prime}$. Clearly, the regions ECK and LHB contribute to the scattering from the gasket material. This contribution results in a high background if boron or boron epoxy gasket is used, and produces diffraction lines if beryllium gasket is used. The assumption that $A B \| C D$ and $\mathrm{EF} \| \mathrm{GH}$ has been made only to define the regions illuminated by the incident beam, and the illuminated region seen by the detector. This is. a very good approximation in view of the small divergences involved in such experiments.

It is possible, by reducing the divergence of the receiving slit, to arrive at a value of $2 b$ for a given set of values of $2 a, 2 \theta_{0}$ and the radius $r$ of the specimen such that the regions ECK and LHB do not exist. Such a situation is shown in figure 3; a region, $\mathrm{CG}^{\prime} \mathrm{BF}^{\prime}$ only of the specimen illuminated by the incident beam is seen by the detector. In such a case the scattering from the gasket material is eliminated. The radiation scattered by the specimen passes through the gasket before reaching the detector, and interacts with the gasket material producing a rescattered radiation which reaches the detector. However, the intensity of the rescattered radiation is very small as compared with the intensity of radiation produced by the scattering of the primary incident beam, and can be neglected. Thus, the diffraction data gathered in the absence of regions ECK and LHB will be practically free from the scattering from the gasket material.

The separation, $2 b_{\max }$, between the lines $\mathrm{CF}^{\prime}$ and $\mathrm{G}^{\prime} \mathrm{B}$ is the maximum value of $2 b$ (for a given set of values of $2 a, 2 \theta_{0}$ and $r$ ) which can lead to the absence of regions ECK and LHB. It is seen from figure 3 that the situation resulting in the absence of the scattering from the gasket material can arise only if $2 \theta_{0}>2 \theta_{\min }$, where $2 \theta_{\min }$ is the angle $\mathrm{ABC}$. Noting that $\mathrm{AC}=2 a, \mathrm{BC}=2 r$, and the angle $\mathrm{CAB}$ is a right angle, $2 \theta_{\min }$ is given by

$$
\sin 2 \theta_{\min }=a / r \text {. }
$$

For $2 \theta_{0}>2 \theta_{\text {min }}$, the relation connecting the various quantities can be obtained by 
considering the triangle $C F^{\prime} B$. Noting that $C F^{\prime}, F^{\prime} B$ and $B C$ equal respectively $2 a / \sin 2 \theta_{0}, 2 b_{\max } / \sin 2 \theta_{0}$ and $2 r$, the following relation can be written,

$$
a^{2}+b_{\max }^{2}+2 a b_{\max } \cos 2 \theta_{0}-r^{2} \sin ^{2} 2 \theta_{0}=0 .
$$

At $2 \theta_{0}=2 \theta_{\min }, 2 b_{\max }=0$. The value of $2 b_{\max }$ increases with increasing value of $2 \theta_{0}$ and reaches a maximum value at $2 \theta_{0}=90^{\circ}$. At $2 \theta_{0}=4 \theta_{\min }, 2 b_{\max }=2 a$.

It is instructive to estimate the numerical values of the various parameters discussed so far. On substituting a reasonable set of values $\left(l_{i}=7.5 \mathrm{~mm}, L_{i}=75 \mathrm{~mm}\right.$ and $W_{i}=0.15 \mathrm{~mm}$ ) for the various quantities in (1) and (2), $\alpha_{i}=2 \mathrm{mrad}$ and $2 a=0.17 \mathrm{~mm}$ are obtained. Assuming $r=0.5 \mathrm{~mm}, 2 \theta_{\min } \simeq 10^{\circ}$ is calculated from (6). In experiments with either molybdenum or tungsten target, $2 \theta_{0}$ is set at $20^{\circ}$. Thus, the condition $2 \theta_{0}>2 \theta_{\min }$ is satisfied. The value of $2 b_{\max }$ as calculated from $(7)$ is $0.16 \mathrm{~mm}$. A diffracted beam collimator with essentially the same dimension as those given above for the incident beam collimator will be required for $2 b_{\max }=0.16 \mathrm{~mm}$. With a fine focus tube, it is possible to further reduce $\alpha_{i}$ without appreciable reduction in the intensity of the incident beam.

\section{Specimen size optimization}

The intensity of the diffracted beam is proportional to the volume of the specimen. However, the larger the size of the specimen, the larger is the reduction in the intensity of the diffracted beam, because of the absorption factor. By considering the specimen volume and absorption factor effects for a geometry required to eliminate gasket scattering it can be shown (appendix) that the diffracted beam intensity reaches a maximum when,

$$
2 a=2 b=-\left(\sin 2 \theta_{0} \ln \cos 2 \theta_{0}\right) / \mu\left(1-\cos 2 \theta_{0}\right) .
$$

where $\mu$ is the linear absorption coefficient of the sample material. Since the absorption coefficient depends strongly on the energy of the radiation, it is not possible to choose a single set of values of $2 a$ and $2 b$ in accordance with (8) which will be valid for the entire energy range. In most cases, the diffraction data are collected in the energy range approximately 10 to $40 \mathrm{keV}$. The optimum values of $2 a$ and $2 h$ may be chosen at $25 \mathrm{keV}$, an energy in the middle of useful energy range. Even this criterion may often lead to $2 a$-value which is unacceptable from other considerations. For example, $r \leqslant 0.5 \mathrm{~mm}$, for the gaskets of $3 \mathrm{~mm}$ dia. Further, from practical consideration $2 a$ and $2 b$ should be in the range $0 \cdot 1-0 \cdot 2 \mathrm{~mm}$. Obviously, the optimum value of $2 a$ obtained from (8) will be unacceptable if it falls grossly outside this range. The optimum value of $2 a$ for low (high) absorbing materials is too large (small) to be achieved in practice. For the optimum value of $2 a$ to be in the range $0 \cdot 1-0.2 \mathrm{~mm}$ at $2 \theta_{0}=20, \mu$-value should be in the range $35 \mathrm{~cm}^{-1}$ to $17.5 \mathrm{~cm}^{-1}$.

The effective absorption coefficient of highly absorbing materials can be brought in the desired range by diluting it with the appropriate proportion of low absorbing material. For example, for niobium $\mu=385 \mathrm{~cm}^{-1}$ at $24.9 \mathrm{keV}$, the corresponding value of $2 a$ from $(8)$ being $0.009 \mathrm{~mm}$. However, diluting niobium powder with sodium chloride in the weight ratio $1: 3$ gives $\mu=35 \mathrm{~cm}^{-1}$. It is fortuitous that sodium chloride is used as a pressure marker. 


\section{Experimental results}

The analysis proposed in this paper was verified by gathering the energy-dispersive data on sodium chloride. A pair of tungsten carbide anvils with the flat face diameter of $3 \mathrm{~mm}$ was used to pressurize the specimen. The experiments were conducted using both boron-epoxy and beryllium gaskets. In either case, the gasket was $3 \mathrm{~mm}$ in diameter and $0.3 \mathrm{~mm}$ thick with a central hole of $1 \mathrm{~mm}$ dia. The X-ray source was a Enraf-Nonius generator with fine focus tungsten target sealed tube operated at $20 \mathrm{~mA}$ and $60 \mathrm{kV}$. The data were collected at $2 \theta_{0}=18^{\circ}$ using a $\mathrm{Si}(\mathrm{Li})$ solid state $\mathrm{X}$ ray detector from Ortec and the associated electronics Ortec 6230: A counting time of $8000 \mathrm{sec}$ was used. First, the data were gathered (with both boron-epoxy and beryllium gaskets) by setting the incident and the diffracted beam divergences such that $2 a=2 b \simeq 0.6 \mathrm{~mm}$. Next, the divergences were reduced such that $2 a=2 b \simeq 0.3 \mathrm{~mm}$ and the experiments repeated.

The data gathered with boron-epoxy gaskets are shown in figure 4 . The inset shows the data with large divergence $(2 a=2 b \simeq 0.6 \mathrm{~mm})$. Only the first two strong peaks are seen on a large background. The data under the condition $2 a=2 b \simeq 0.3 \mathrm{~mm}$ clearly show the first five strong peaks of sodium chloride on a relatively low general background. The data with beryllium gasket under the condition $2 a=2 b \simeq 0.6 \mathrm{~mm}$ are shown in the inset of figure 5. The strong beryllium lines are seen together with the sodium chloride lines. In practice the interference from the beryllium lines can pose serious problems. The reduction of the divergences of the incident and the diffracted beams results in a considerable improvement of the diffraction patterns. The intensity of the beryllium line drops considerably. Ideally, the beryllium lines should be absent. However, in figure 5, (101) beryllium line appears with a very low intensity presumably because of small misalignment.

\section{Discussion}

The tungsten carbide opposed anvil set-up was extensively used in sixties and early seventies in high pressure $x$-ray diffraction studies. The gasket made from

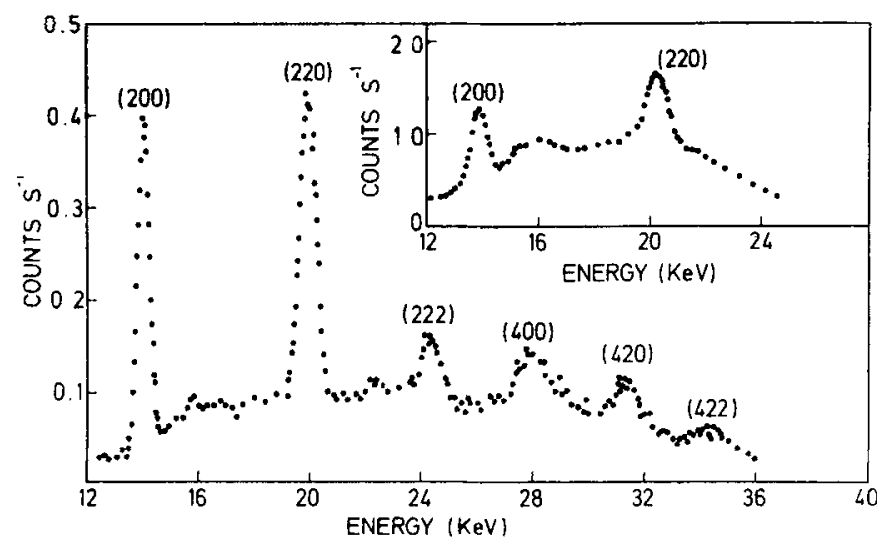

Figure 4. Sodium chloride data recorded with boron-epoxy gasket under the conditions $2 a=2 b \simeq 0.3 \mathrm{~mm}$, and $2 \theta_{0}=18$. The inset shows the data recorded with $2 a=2 b \simeq 0.6 \mathrm{~mm}$. 


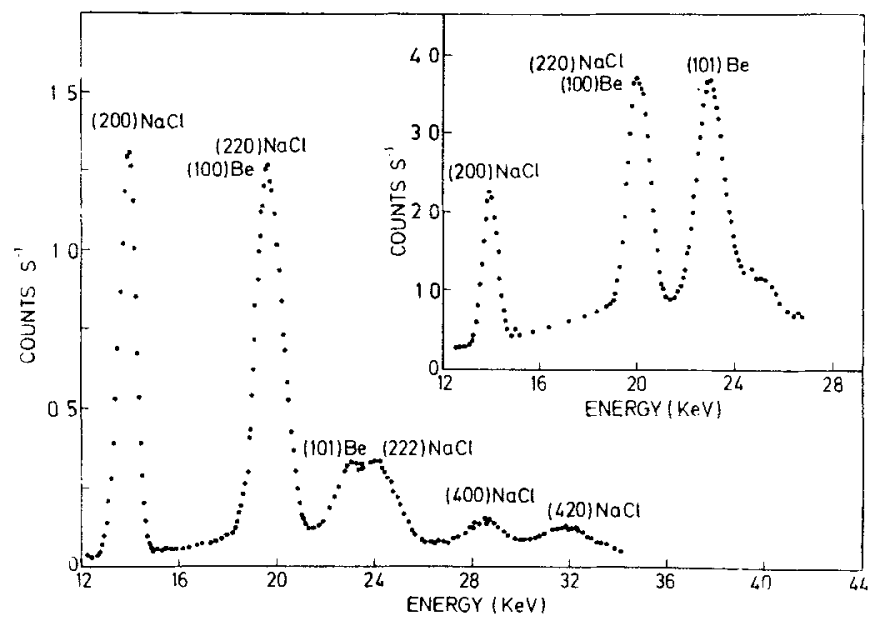

Figure 5. Sodium chloride data recorded with beryllum gasket under the conditions $2 a=2 b=0 \cdot 3 \mathrm{~mm}$. and $2 \theta_{0}=18$. The inset shows the data recorded with $2 a=2 b \simeq 0 \cdot 6 \mathrm{~mm}$.

amorphous boron (or amorphous boron and epoxy mixture) was used to contain the specimen. The set-up was inexpensive and extremely easy to operate. It provided a good control over the small pressure increments. The method, however, suffered from two major drawbacks. Firstly, the pressure on the specimen was not truly hydrostatic, and this introduced systematic errors in the pressure-volume curve derived from X-ray measurements (Singh 1978). Secondly, the X-ray scattering from the gasket material produced high background. These drawbacks outweighed the advantages of lower cost and ease of operation, and this method was discarded in favour of other methods such as diamond anvil cell.

Halleck and Olinger (1974) solved the problems arising from the non-hydrostatic pressure distribution on the specimen by replacing boron gaskets by beryllium gaskets. The use of beryllium gaskets made it possible to seal fluid together with the specimen and generate truly hydrostatic pressure. This proved to be an important improvement in the tungsten carbide opposed anvil technique. The background scattering, however, did not decrease appreciably with the use of beryllium gaskets. The diffraction lines from beryllium gasket added further complications.

The present analysis of the Freud-LaMori geometry indicates that a proper choice of the divergences of the incident and diffracted beams can lead to a situation wherein the radiation scattered from the gasket material does not enter the detector. This results in an improved peak-to-background ratio if boron-epoxy gasket is used, and eliminates unwanted diffraction lines from beryllium, if beryllium gaskets are used.

It may be noted that any degree of collimation cannot give this result if photographic film is used to record the diffraction pattern. In principle, angle dispersive method with a scintillation or a proportional counter detector can give this result at $0>\theta_{\min }$. However, the use of a scintillation or a proportional counter is not practicable because of the extremely low count rate produced by the diffracted beam in angle dispersive mode. The use of the energy-dispersive method is crucial in realizing in practice the geometry discussed in this paper. 


\section{Conclusion}

The present analysis of Freud-LaMori geometry and the data collected under these conditions clearly indicate that the scattering from the gasket material reaching the detector can be eliminated by suitably limiting the divergences of the incident and the diffracted beams. Thus, the use of beryllium gasket with fluid pressure transmitter, and Freud-LaMori geometry for data collection using energy dispersive method eliminates both the major drawbacks of the tungsten carbide opposed anvil set-up. It is hoped that with these improvements, the tungsten carbide opposed anvil set-up will prove to be a reliable method of recording X-ray diffraction data from the specimen pressurized up to $15 \mathrm{GPa}$.

\section{Acknowledgement}

The authors thank Dr R V Krishnan for many helpful discussions.

\section{Appendix}

Let the $x$ - and $y$-axes be respectively along $O U$ and $O V$ (figure 6). Clearly,

$$
\begin{aligned}
& O U(\equiv 2 \bar{a})=2 a / \sin 2 \theta_{0}, \\
& O V(\equiv 2 \bar{b})=2 b / \sin 2 \theta_{0} .
\end{aligned}
$$

Consider an area element $\mathrm{d} x \mathrm{~d} y$, at $N$ with coordinates $(x, y)$. The incident ray $L^{\prime} N$, is scattered by element $(\mathrm{d} x \mathrm{~d} y)$ along $N P^{\prime}$ parallel to $x$-axis. Neglecting small path lengths $L^{\prime} L$ and $\mathrm{PP}^{\prime}$, the total path length, $l$, in the specimen is given by,

$$
\begin{aligned}
l & =L N+N P ;=(L M+M N)+(Q S-Q N+S P) ; \\
& =\left(x \cos 2 \theta_{0}+y\right)+\left(2 \bar{a}-x+S T \cos 2 \theta_{0}\right) ; \\
& =\left(x \cos 2 \theta_{0}+y\right)+(2 \bar{a}-x)+(U T-U S) \cos 2 \theta_{0} ; \\
& =2 \bar{a}+2 \bar{b} \cos 2 \theta_{0}-(x-y)\left(1-\cos 2 \theta_{0}\right) .
\end{aligned}
$$

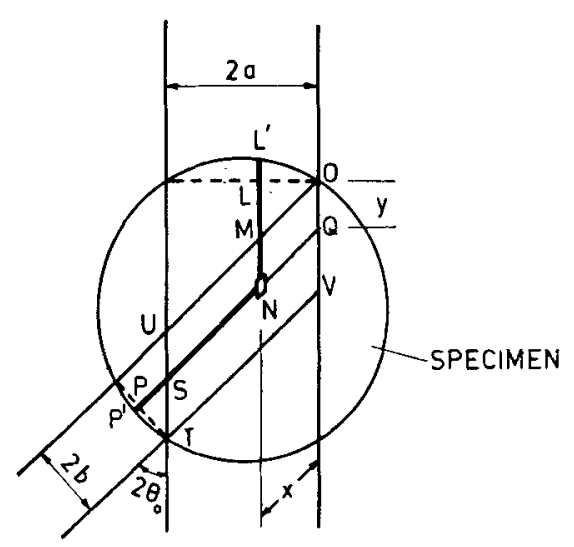

Figure 6. The $\mathrm{X}$-ray beam path length in the specimen. 


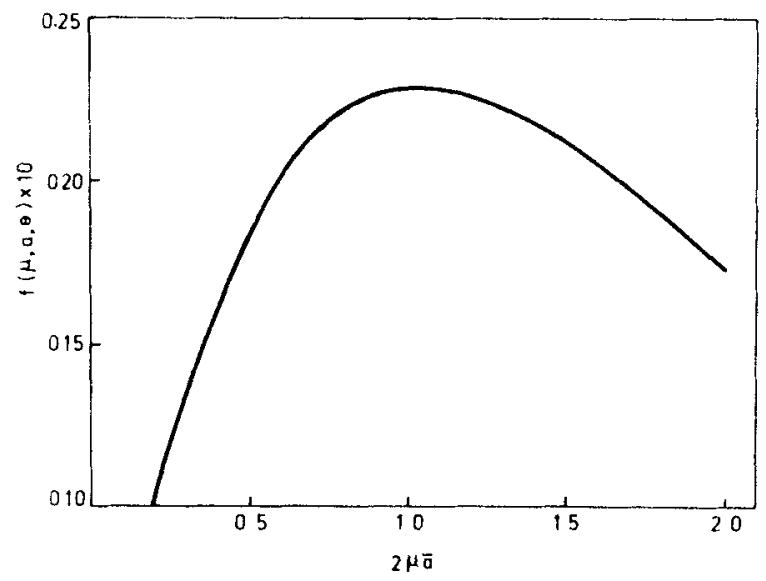

Figure 7. A plot of $f(\mu, \bar{a}, \theta)$ vs $2 \mu \bar{a}$.

In deriving (11), the following equalities in addition to (9) and (10) have been used;

$$
\begin{aligned}
& Q S=O U=2 \bar{a} ; U T=O V=2 \bar{b} ; \\
& O Q=M N=U S=y ; Q N=O M=x .
\end{aligned}
$$

The intensity of the radiation scattered from $(\mathrm{d} x \mathrm{~d} y)$ along $N P^{\prime}$ is proportional to the volume element ( $\mathrm{d} x \mathrm{~d} y) t$ (where $t$ is the thickness of the cylindrical specimen) and $\exp (-\mu l)$. The total intensity reaching the detector is given by

$$
I=C \int_{0}^{2 \bar{a}} \int_{0}^{2 \bar{b}}(\exp -\mu l) \mathrm{d} x \mathrm{~d} y
$$

On substituting for I from (11) and integrating, the following expression is obtained,

where

$$
I=\left[K / \mu^{2}\left(1-\cos 2 \theta_{0}\right)^{2}\right] f\left(\bar{a}, \mu, \theta_{0}\right) f\left(\bar{h}, \mu, \theta_{0}\right)
$$

$$
f\left(\bar{a}, \mu, \theta_{0}\right)=\exp \left(-2 \mu \bar{a} \cos 2 \theta_{0}\right)-\exp (-2 \mu \bar{a}) .
$$

It can be easily shown that $I$ reaches a maximum value when,

$$
2 \bar{a}=2 \bar{b}=-\ln \left(\cos 2 \theta_{0}\right) / \mu\left(1-\cos 2 \theta_{0}\right)
$$

The variation of $f(\bar{a}, \mu, \theta)$ with $\bar{a} \mu$ is shown in figure 7 .

\section{References}

Freud P J and LaMori P N 1969 Trans. Am. Crystallogr. Assoc. 5155

Halleck P M and Olinger B 1974 Rer. Sci. Instrum. 451408

Jamieson J C and Lawson A W 1962 J. Appl. Phys. 33776

McWhan D B and Bond W L. 1964 Rer. Sci. Instrum. 35626

Perez-Albuerne F. A. Forsgren K F and Drickamer H G 1964 Rev. Sci. Instrum. 3529

Singh A K 1978 liigh Temp-High Pressures 10641 